\title{
Development of Thick Intrinsic Silicon Detectors for Hard X-ray and Gamma-ray Detection
}

\author{
B. F. Phlips, W. N. Johnson, R. A. Kroeger, J. D. Kurfess
}

\begin{abstract}
We are developing thick intrinsic silicon detectors for hard X-ray and gamma-ray detection. The goal is to manufacture detectors with good spectroscopy and imaging capabilities without the need for cryogenics. Our current baseline design is a double-sided silicon strip detector with an active area of $\sim 60 \mathrm{~mm} \times 60 \mathrm{~mm}$ and a thickness of $2 \mathrm{~mm}$. To demonstrate the feasibility of $2 \mathrm{~mm}$ thick intrinsic detectors, we tested a $2 \mathrm{~mm}$ thick single pixel detector. An energy resolution of $1 \mathrm{keV}$ FWHM at $60 \mathrm{keV}$ was achieved at an operating temperature of $-65 \mathrm{C}$. The detector was fully depleted at $\sim 800 \mathrm{~V}$. We present the detailed results of tests on the pixel detector, the design of our double sided detector, and the conceptual design of hard X-ray and gamma ray instruments that use these detectors.
\end{abstract}

\section{INTRODUCTION}

Detectors for high resolution gamma-ray spectroscopy have traditionally been made of germanium or lithium-doped silicon [1]. These materials both require operation at liquid nitrogen temperature, and the detectors need to be handcrafted. Recently, Cadmium Telluride (CdTe) and Cadmium Zinc Telluride ( $\mathrm{CdZnTe}$ ) were introduced as room temperature semi-conducting detectors. While a lot of progress has been made in the development of these detectors [2], [3], they are still not available in large sizes and the manufacturing processes still need to be industrialized. Intrinsic silicon, on the other hand, has a large industrial base but has only seen limited application as a gamma ray detector. This is due to the fact that most intrinsic silicon detectors are 300 micrometers thick (the standard thickness for the semi-conducting industry) and therefore have a very low efficiency for stopping gamma rays. Thicker intrinsic silicon detectors can be made, but for the same material, the depletion voltage increases as the square of the thickness. Detectors with a thickness of $2 \mathrm{~mm}$

Manuscript received November 2, 2001. This work was supported in part by the U.S. Defense Threat Reduction Agency (DTRA) and the US National Aeronautics and Space Administration (NASA).

B. F. Phlips is with the Naval Research Laboratory, Washington DC 20375 USA (telephone: 202-767-3572, e-mail: phlips @gamma.nrl.navy.mil).

W. N. Johnson is with the Naval Research Laboratory, Washington DC 20375 USA (telephone: 202-767-6817, e-mail: johnson @ gamma.nrl.navy.mil).

R. A. Kroeger is with the Naval Research Laboratory, Washington DC 20375 USA (telephone: 202-404-7878, e-mail: kroeger@gamma.nrl.navy.mil).

J. D. Kurfess is with the Naval Research Laboratory, Washington DC 20375 USA (telephone: 202-767-3182, e-mail: kurfess @ gamma.nrl.navy.mil). would then require a depletion voltage of a few thousand volts. Using float zone refining, high purity silicon with a resistivity of $30000 \mathrm{Ohm}-\mathrm{cm}$ is now available [4], compared to a resistivity of $\sim 2000 \mathrm{Ohm}-\mathrm{cm}$ typical of thin strip detectors. A depletion voltage of less than $1000 \mathrm{~V}$ is required with this material, making the development of detectors more feasible. The design of the edge of the detector still requires great care to be able to handle the high voltage. While designs with a single guard ring have been made to work [5], a common approach is to use multiple guard rings.

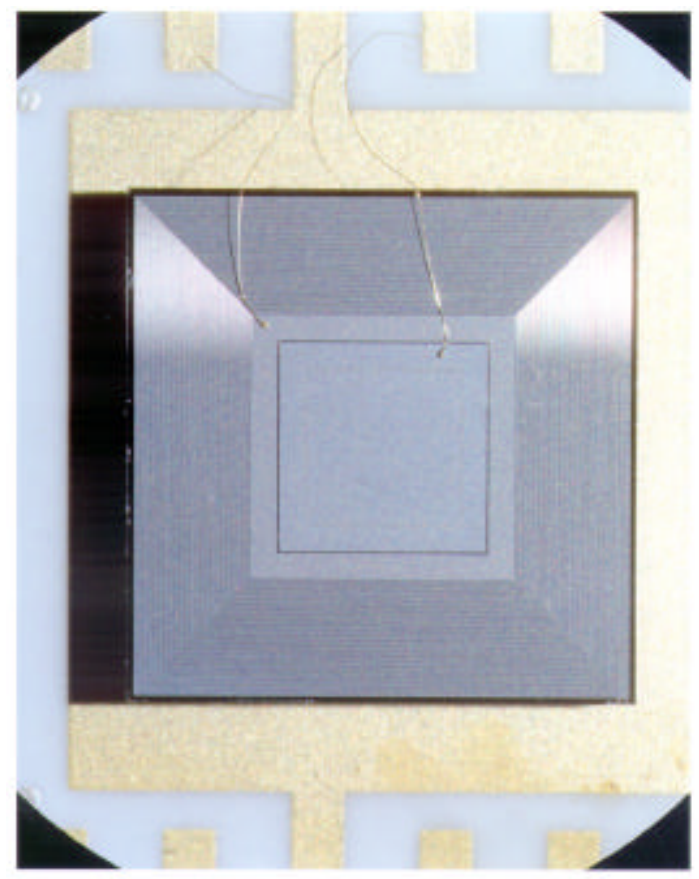

Fig. 1. Top view of the thick silicon pixel detector. The total detector area is $10 \times 10 \mathrm{~mm}$. The active area is $4 \times 4 \mathrm{~mm}$.

We have started a program to develop thick (2 mm) intrinsic detectors for X-ray and gamma ray detection. The first step is to study the noise performance of a single pixel and its guard structure. The guard structure is independent of the active part of the detector and can therefore best be tested with single pixel detectors. Results are shown in section II. The next step in our development effort is the production of double-sided strip detectors. Section III will outline our detector design. An alternative approach to strip detectors is to develop detectors with arrays of pixels and 
drift detectors. This approach is being pursued by other groups and has shown very promising results [6]. However, there are applications where a very large number of detectors are needed and very little passive material is allowed. For such applications, strip detectors are more suitable than pixel detectors. Section IV outlines two such proposed instruments.

\section{PROTOTYPe Pixel Detector}

To demonstrate the feasibility of $2 \mathrm{~mm}$ thick intrinsic detectors, we tested the performance of a single pixel, $2 \mathrm{~mm}$ thick intrinsic detector. The detector was manufactured by SINTEF Electronics and Cybernetics [7]. The detector has an area of 10x10 mm, with an active area of $4 \times 4 \mathrm{~mm}$ (see Fig. 1.). The detector has multiple (50) concentric guard rings to withstand the large voltages needed to deplete $2 \mathrm{~mm}$ of silicon (see Fig. 2). A similar guard ring structure will be used on the double-sided detector. While these structures obviously take up a large fraction of the effective area for a pixel detector, they will only take up $\sim 18 \%$ of the effective area for the $60 \mathrm{~mm} \times 60 \mathrm{~mm}$ double sided strip detector, and would take up only $12 \%$ of the area for $95 \mathrm{~mm}$ x $95 \mathrm{~mm}$ detectors possible from six inch material.

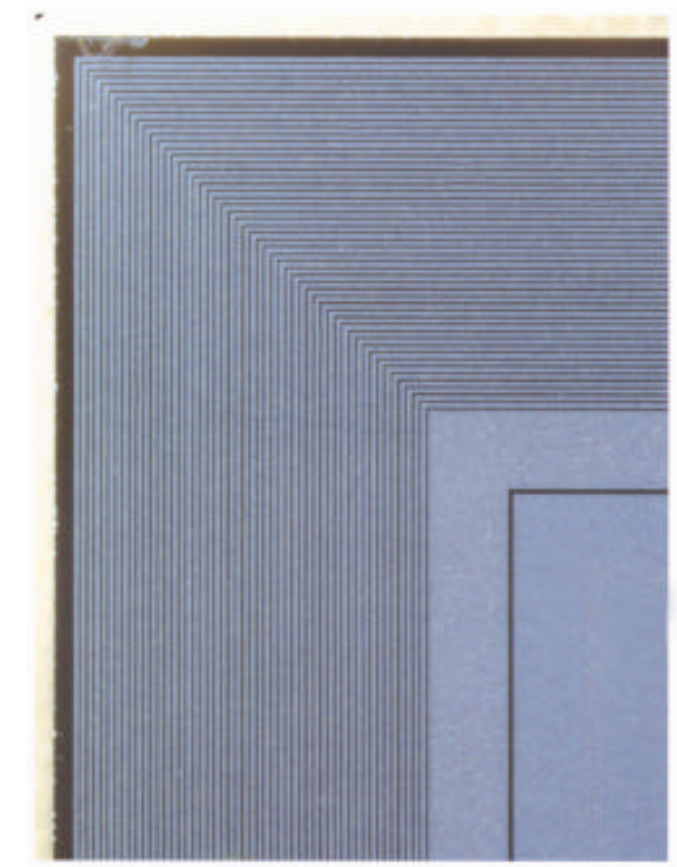

Fig. 2. Detailed view of the corner of the pixel detector. Note the multiple guard ring structures.

Although this pixel detector was originally designed for heavy-ion detection, we tested its performance as a hard Xray detector. We illuminated the detector with a sealed Americium-241 source. The resulting spectrum is shown in Fig. 3. The spectrum was obtained using a hybrid preamplifier (eV Products 5093) [8], a NIM shaping amplifier, and a NIM-based ADC. The shaping time was 1 microsecond. The detector was fully depleted at $\sim 800 \mathrm{~V}$. The applied voltage of $900 \mathrm{~V}$ generated a leakage current of less than $100 \mathrm{pA}$ at a temperature of $\sim-40 \mathrm{C}$. At a temperature of $-65 \mathrm{C}$, the energy resolution was $1.0 \mathrm{keV}$ FWHM for the $60 \mathrm{keV}$ line. The low energy events in the broad continuum below the $60 \mathrm{keV}$ line are due to interaction in an aluminum lid between the source and the detector. At this temperature, the energy resolution is dominated by the performance of the front-end electronics. We plan further tests with lower noise electronics to probe the intrinsic detector performance. At $0 \mathrm{C}$, the leakage current was still only $\sim 1 \mathrm{nA}$, demonstrating the sturdiness of the design. At $0 \mathrm{C}$, the spectral resolution was slightly

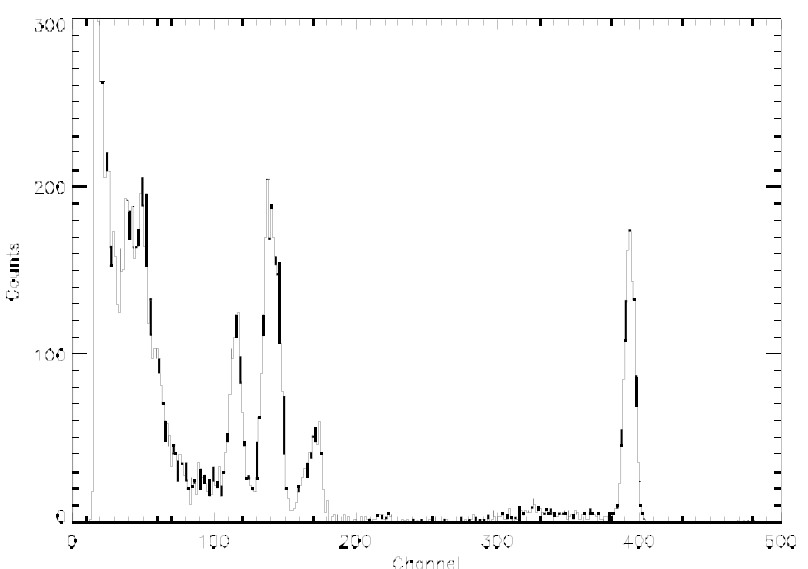

Fig. 3. Spectrum of Am-241 source as measured with the thick silicon detector. The $60 \mathrm{keV}$ peak is near channel 400.

degraded due to the increased leakage current.

\section{DOUBLE SIDED STRIP DETECTOR}

We are currently designing a $2 \mathrm{~mm}$ thick double-sided orthogonal strip detector with $\sim 63 \mathrm{~mm}$ x $63 \mathrm{~mm}$ area and an active area of $57 \mathrm{~mm} \times 57 \mathrm{~mm}$ (Fig. 4). The material for the detector is high-resistivity silicon (>20000 Ohm cm) extracted from 4 inch float-zone boules. The guard structure is similar to the one shown in Fig.2. The multiple guard rings are not held at a specific but float to their respective voltages to generate a smooth voltage gradient. The depletion voltage is expected to be $\sim 800$ volts. The strip pitch is $\sim 0.89 \mathrm{~mm}$, so that the device has $64 \times 64$ channels. The strips will be DC coupled to keep the design simple and reliable. Surface mount de-coupling capacitors will be used on the readout board for the high voltage side signals. The expected capacitance for a strip to the backplane is expected to be $\sim 0.4 \mathrm{pF} / \mathrm{cm}$, and the total capacitance is expected to be $\sim 2 \mathrm{pF} / \mathrm{cm}$. The resistance of the aluminum metalization on the strips is kept to a low $\sim 1 \mathrm{Ohm} / \mathrm{cm}$ to minimize the noise contribution of this resistance. The strips will have bond pads at either end of the strips to allow the daisy-chaining of multiple detectors. The leakage current is expected to be less than $0.5 \mathrm{nA}$ per strip at $-40 \mathrm{C}$. The planned capacitance and leakage current should allow a spectroscopic performance of better than $1 \mathrm{keV}$ for hard X-rays. 
The design of the detector is almost finished and the first detectors should be ready in March 2002.

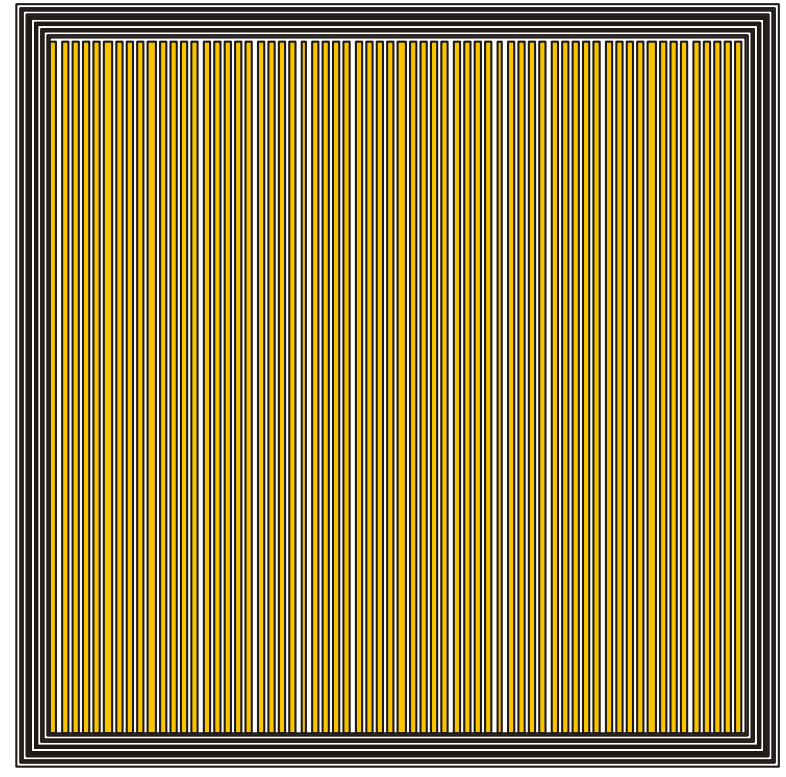

Fig. 4. Design of the double-sided $2 \mathrm{~mm}$ thick detector. The detectors are $63 \mathrm{~mm} \times 63 \mathrm{~mm}$ in size, with an active area of $57 \mathrm{~mm} \times 57$ $\mathrm{mm}$, and 64 strips per side. The guard ring structure is similar to the one shown in Fig. 2.

\section{APPLICATIONS}

There are many potential applications for X-ray detectors with imaging and spectroscopy, from material properties studies using X-ray fluorescence to medical research [9]. We will focus here on two applications of interest to our sponsors: the detection of hard $\mathrm{x}$-rays at the focal plane of an X-ray telescope and an Advanced Compton Telescope for the detection of nuclear lines.

\section{A. Hard X-Ray Focal Plane Instrument}

NASA's proposed Constellation-X mission [10] will include a Hard X-ray Telescope that will extend the powerful technique of focusing optics beyond $10 \mathrm{keV}$. At these higher energies, an individual silicon CCD can no longer stop the focused X-rays. New focal plane instruments are therefore needed. These instruments only need moderate position resolution ( a few tenths of a millimeter) but good energy resolution (a fraction of a keV) and low internal background. A stack of silicon strip detectors (see Fig. 5) would provide an excellent focal plane instrument for HXT. At low energies (below $10 \mathrm{keV}$ ), only the frontal detector needs to be used and therefore only its small mass contributes to internal background. At higher energies (10$40 \mathrm{keV}$ ), the interaction is still mostly photoelectric and again only a single detector is typically involved with an event. If multiple detectors are involved in an event, then the geometry of the interaction locations must be consistent with Compton kinematics to be a valid event. This kinematic reconstruction requirement is a powerful background rejection technique. A stack of 10 silicon detectors $\mathrm{f} 2 \mathrm{~mm}$ thickness would fully absorb all photons up to $\sim 40 \mathrm{keV}$.

Silicon detectors are a more mature and space-qualified technology than the proposed CdTe or CdZnTe alternatives. Silicon will undergo less radioactive activation than other materials when exposed to the cosmic ray environment in space. Silicon also has the advantage of having a smooth
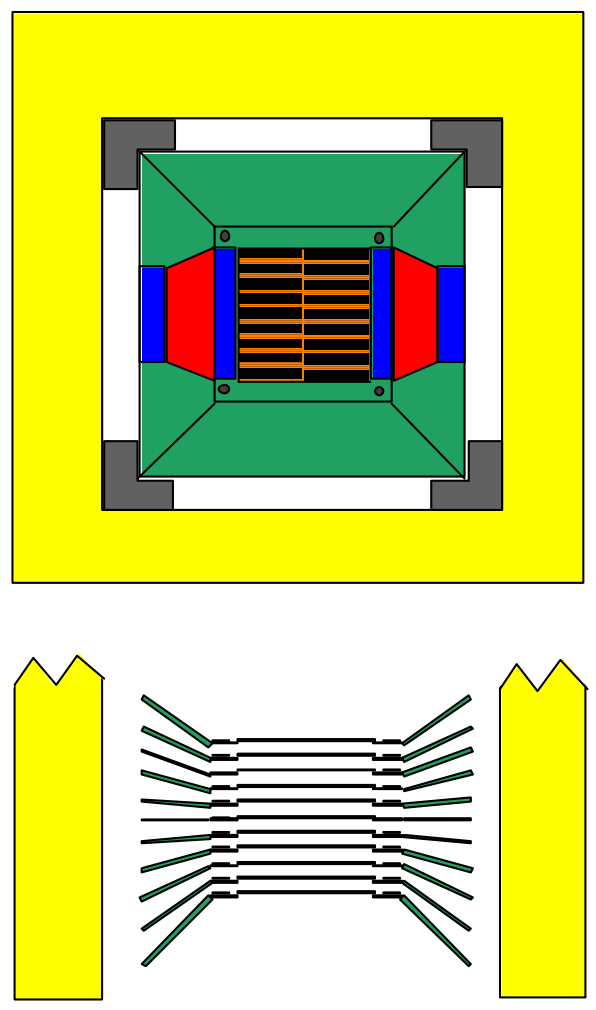

Fig. 5. Conceptual drawing of a focal plane instrument. The top drawing is a top view of a detector plane. (The opposite side of the same detector plane would have the strips and readout orthogonal to those shown in the top view.) The bottom drawing is a side view. The height of the shielding has been truncated in the bottom view. energy response over the energy range of interest: there are no absorption edges or $\mathrm{K}$-escape photons in the entire range of the instrument, unlike CdTe or CdZnTe.

\section{B. Advanced Compton Telescope}

The Advanced Compton Telescope (Fig. 6) is a proposed next generation Compton telescope. It would be sensitive from a few hundred $\mathrm{keV}$ to tens of $\mathrm{MeV}$. It would consist entirely of semi-conducting (silicon) position sensitive detectors. It would be capable of spectroscopy on all strips. It would be $\sim 120 \mathrm{~cm} \times 120 \mathrm{~cm}$ in area and $85 \mathrm{~cm}$ deep. The implementation shown in Fig. 6 consists of 16 identical vertical stacks or towers. Each tower consists of 24 layers of detectors. Each layer (Fig. 7) consists of a $4 \times 4$ array of detectors. The layers are held in a vertical stack by cooling pipes. The instrument would operate at $\sim-40 \mathrm{C}$. The entire instrument would contain $\sim 6100$ double-sided silicon strip detectors and have $~ 200000$ channels of spectroscopy. Since spectroscopy is required on all strips, power 
dissipation constraints will require the daisy-chaining of multiple strip detectors together. Assuming $2 \mathrm{~mW}$ of low power CMOS electronics per channel, a front-end power of $>400 \mathrm{~W}$ would still be required. The plane of detectors shown in Fig. 7 would consist of a $4 \times 4$ array of detectors edge bonded to each other with epoxy. The strips on one side of the detectors would be electronically daisy-chained in one direction with wire bonds, and the other side would be daisy-chained in the orthogonal direction. The $4 \times 4$ detector array would be self-supporting and only be supported around its perimeter.

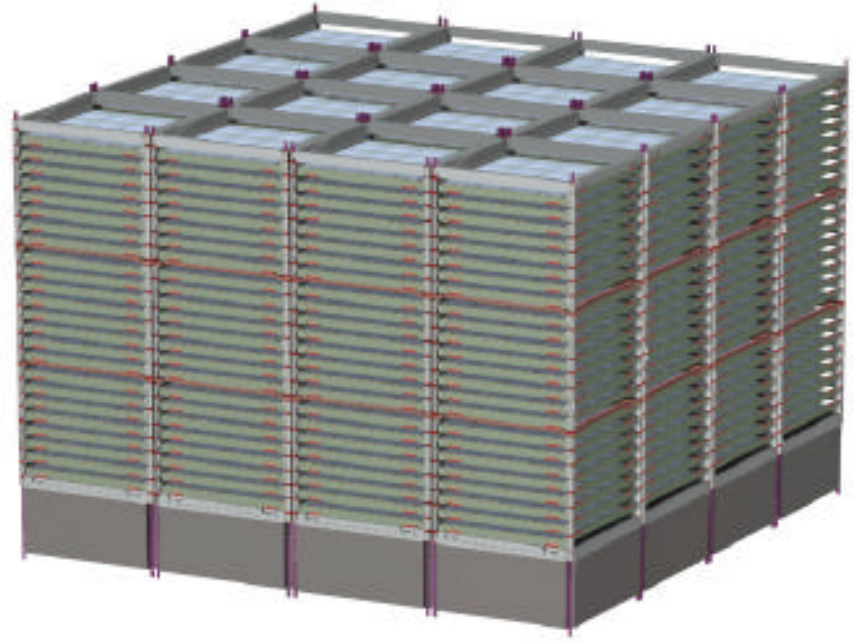

Fig. 6. Design of an Advanced Compton Telescope consisting of 16 detector towers. Each tower consists of 24 planes of silicon detectors. Each plane consists of 16 silicon detectors tiled into a $4 \times 4$ array. Electronics are mounted between the silicon layers and cooling tubes run vertically in the corners of each tower.

There are multiple reasons for constructing an Advanced Compton Telescope out of silicon. Using silicon detectors, not cryogenics are required, unlike a germanium array. While manufacturing $20 \mathrm{~m}^{2}$ of silicon detectors is a daunting task, it will be substantially more practical to manufacture than a similar quantity of CdTe or CdZnTe. A more subtle reason for using silicon is that the average electron energy in silicon is lower than in elements with a higher atomic number. The electron energy in the detector is important because it provides a physical limit to the accuracy of the Compton reconstruction. The energy of the electron causes a Doppler broadening of the energy and angle of a Compton scattered photon. The intrinsic energy and angular resolution of a Compton telescope is therefore limited by this electron energy [11].

A crucial aspect of the mechanical design of this instrument is the amount of passive material inside the active volume of the detector. Since a $1 \mathrm{MeV}$ photon will interact $\sim 10$ times in silicon before being fully absorbed, it is important that most of these interactions happen in active detector material. The design shown in Fig. 6 is the result of study that demonstrated a mechanical and thermal structure whose mass is only $10 \%$ of the total mass inside the active volume. Another $10 \%$ of the mass is required for passive silicon in the guard rings of each detector, and an additional $5 \%$ of the mass will be taken up by the electronics. Kurfess et al. [11] have shown that an efficiency of $>30 \%$ was achievable with such a configuration.

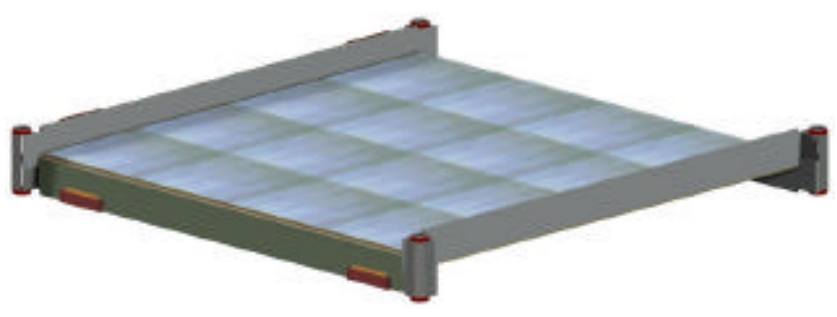

Fig. 7. Individual "Tray" of an Advanced Compton Telescope. Each tray consists of an array of $4 \times 4$ double-sided strip detector that are edgebonded with epoxy and whose strips are daisy-chained by wire bonds. The silicon itself is the horizontal structural member. The front-end electronics are mounted vertically between layers of silicon. The cooling is provided through corner cooling tubes.

A careful study $[12,13]$ of events that undergo multiple interactions inside a position-sensitive spectroscopy detector demonstrates that only the first three interactions are important. By multiple use of the Compton formula, the first three interactions can fully determine the energy of the incoming gamma ray. The energy of a gamma ray can be determined without absorbing it as long as the first two interactions are Compton scatters and the third interaction is also in the active material. An additional implicit requirement is that the correct order of these interactions be determined. Kroeger et al. [14] demonstrates an 86\% efficiency in ordering the interactions for $2.6 \mathrm{MeV}$ photons with 4 interactions. The low atomic number of silicon is actually an advantage in this technique since the ratio of the Compton scattering cross section to the to photo-electric cross section is better than for most detector materials.

\section{Conclusions}

Thick intrinsic silicon detectors have many applications for the ground- and space-based detection of hard X-rays and gamma rays. Detectors are now becoming available for testing and the time to start considering instrument configurations has arrived.

\section{ACKNOWLEDGMENT}

We thank E. Simson for his help in the laboratory at NRL, G. Bredholt of SINTEF electronics and Cybernetics for use of the first pixel detector used in this work, and E. Ponslet at Hytec for his efforts on the mechanical study of the ACT.

\section{REFERENCES}

[1] G. F. Knoll, Radiation Detection and Measurement, $2^{\text {nd }}$ ed., New York: Wiley and Sons, 1989.

[2] T. H. Prettyman, M. C. Browne, K. D. Ianakiev, C. E. Moss and S. E. Soldner, "Characterization of a large volume multi-element CdZnTe detector”, Proc. SPIE, vol. 4141, pp. 1-10, 2000. 
[3] O. Peyret, "Main advances in X-and gamma-ray imaging with CdTe/CZT" submitted for publication, 2001 IEEE Trans. Nucl. Sci.

[4] Topsil Semiconductor Material A/S, 4 Linderupvej, DK-3600 Frederikssund, Denmark.

[5] M. Sugiho, T. Kamae, K. Makishima, T. Takahashi, T. Murakami, M. Tashiro et al., "Spatially dependent response of thick and large area p-In diode for ASTRO-E hard X-ray detector", IEEE Trans. Nucl. Sci., vol. 48, pp. 426-430, 2001.

[6] L. Struder, R. Hartmann, S. Kemmer, N. Krause, D. Stotter, G. Lutz et al., "Room temperature X-and gamma-ray spectroscopy with silicon drift detectors", Proc. SPIE, vol. 4141, pp. 29-47, 2000.

[7] SINTEF Electronics and Cybernetics, PB 124 Blindern, N0314 Oslo, Norway.

[8] eVproducts, 373 Saxonburg Blvd., Saxonburg PA 16056.

[9] J. D. Kurfess and B. F. Phlips, "Coincident Compton nuclear medical imager”, submitted for publication, 2001 IEEE Trans. Nucl. Sci.

[10] http://constellation.gsfc.nasa.gov.

[11] Y. F.Du, Z. He, G. F. Knoll, D. K. Wehe, W. Li, Proc. SPIE, vol. 3768, pp. 228-238, 1999.

[12] J. D. Kurfess, W. N. Johnson, R. A. Kroeger and B. F. Phlips, "Considerations for the next Compton telescope mission", AIP Conf. Proc. 510, pp. 789-793, 2000.

[13] N. Dogan and D. K. Wehe, "Optimization and angular resolution calculations for a multiple Compton scatter camera”, 1993 IEEE-NSS Conference Record, pp. 269-273, 1994.

[14] R. A. Kroeger, W. N. Johnson, J. D. Kurfess, B. F. Phlips and E. A. Wulf, "Three-Compton telescope: theory, simulations, and performance", submitted for publication, 2001 IEEE Trans. Nucl. Sci. 